\title{
Determinants of dietary adequacy among school age children in Guraghe Zone, Southern Ethiopia
}

\author{
Abdu Oumer ${ }^{1}$, Berhanu Abebaw ${ }^{2}$ \\ ${ }^{1}$ Department of Public Health, Wolkite University, Ethiopia \\ ${ }^{2}$ Department of Public Health, Bahr Dar University, Ethiopia
}

\section{Article Info \\ Article history: \\ Received Feb 20, 2019 \\ Revised Mar 29, 2019 \\ Accepted Apr 12, 2019}

\section{Keywords:}

Dietary diversity

Guraghe zone

School age children

\begin{abstract}
Dietary diversity (DD) is a validated proxy indicator of micronutrient adequacy among different age groups including infants, children and women. This study assessed level of dietary adequacy and its associated factors among school age children in Guraghe Zone, Ethiopia. Survey was conducted among 769 children aged 6 to 12 years of with their care givers using multistage sampling method. Data were collected by using structured questionnaire containing the ten food groups for minimum dietary diversity for women and other parts. Adequate dietary diversity was categorized those children who consume at least five food groups. Bivariate and multivariable binary logistic regression with odds ratios $(95 \% \mathrm{CI})$ was computed. Overall 769 children were included in the study, with a mean age of 8 years. The mean dietary diversity score was $4.9( \pm 1.42)$. About $444(58.3 \%)$ had an inadequate dietary diversity. Those children from extended family size had 1.3 times to have inadequate DD level $(\mathrm{AOR}=1.3)$. Children from female headed households, did not attend formal education had 1.3 and 1.4 times higher odds of having an inadequate DD level (AOR=1.3 and 1.4). Similarly, children living with uneducated caregiver had six fold more likely to have an adequate DD level (AOR=6.7). The dietary diversity of children in the study area was below average. Household head, caregiver's educational status, occupation of the household head, father/female headed household and family size were found to be associated with DD score. There should be awareness creation through existing Health extension platform and back yard vegetation should be improved.
\end{abstract}

Copyright $\odot 2019$ Institute of Advanced Engineering and Science. All rights reserved.

\section{Corresponding Author:}

Abdu Oumer,

Department of Public Health,

Wolkite University, Ethiopia.

Email: phnabu@gmail.com

\section{INTRODUCTION}

Globally, an estimated 815 million people suffer from hunger and its related problems like malnutrition. However, malnutrition is beyond less amount of daily energy intake, including micronutrient deficiencies and overweight. School age is a critical period in which the dietary habits of children are formed and learned, knowing the predictors of diversified diet intake is crucial [1]. Micronutrient deficiencies are more prevalent among school age children with great devastating effects. The multiple micronutrient deficiency was reported in about $79.5 \%$ of children while about $40 \%$ had one micronutrient deficiency, including Zinc, vitamin A, iron and others [2]. Reports from different part of the country showed that malnutrition is a common problem affecting a significant number of school age children. The prevalence estimates of stunting and thinness ranges from $11.52 \%$ to $42.7 \%$ and from $15.7 \%$ to $58.3 \%$ respectively [3-5]. Malnutrition among children has been associated with higher risks of morbidity, mortality, decreased cognitive capacity, blindness, decreased school performance and increased class misses [6]. 
Dietary diversity (DD) is a validated proxy indicator of micronutrient adequacy among different age groups, including infants, young children and women. The Food and Nutrition Technical Assistance (FANTA) group and Agricultural Organization has developed a different DD scale for children and omen [7]. The higher DD score is positively correlated related with different nutritional indicators like weight for age and height for age [8]. Similarly, increased intake of diversified diet has been linked to lower risk of Micronutrient deficiencies [2]. Similarly, world Health Organization (WHO) recommends plant and animal based diversified diet intake to alleviate micronutrient deficiency [6].

For younger children FAO developed a validated seven group DD score that can be scored out of seven. Since the dietary habits of older children, especially school ages is different and completely relies on dietary sources of energy than breast milk, the ten food group DD score out of ten over performs in assessing DD among older children [7]. DD score is a strong predictor of different micronutrient deficiencies of public health importance among children in Ethiopia [9]. Other studies also showed that higher DD score was significantly associated with micronutrient adequacy $(\mathrm{P}<0.0001)$ and improved linear growth at different stages of life $(\mathrm{P} \leq 0.028)$ [10]. It is one of the predictor for nutritional status among children. Thus DD score is a simple, useful tool applicable for the community to identify early risks of malnutrition [11]. It is affected by the seasons of assessment, in that the mean DD score was found to be higher in rainy season $(\mathrm{P}<0.001)[12]$. Studies from different area showed that dietary intake of varied food is limited, especially animal source foods [13-14].

Knowing that malnutrition is common among school age children in different parts of the country [3-5], and dietary diversity is easy to administer and validated tool for early assessment of micronutrient adequacy and early nutritional risk among children [2,11]. Hence it is imperative and useful to conduct this study in order to assess the magnitude of problem and indirectly understanding nutrition insecurity within this age group. This study assessed the dietary adequacy level and its correlates among children aged 6 to 12 years of age in southern Ethiopia.

\section{RESEARCH METHOD}

This study was conducted in Guraghe zone, which is one of the administrative zones in Southern Nations and Nationalist Region. It has 13 districts (Abeshegie, Cheha, Endegagn, Enemorina Eaner, Ezha, Geta, Gumer, Kebena, Gedebano Gutazer Welene, Mareko, Meskane, Muhor Aklil and Soddo woredas) and two town administrations (Butajira and Wolkite town). Wolkite town is the capital of Guraghe zone, which is found $425 \mathrm{~km}$ and $153 \mathrm{~km}$ from Hawasa and Addis Ababa respectively on the way to Jimma. Abeshege, Cheha, Enemorina Eaner, Meskan and Mareko district, has a total population of 61,424, 115,951, $167,770,155,782$ and 64,512.

Community based cross sectional study was conducted among randomly selected school age children aged from 6 to 12 years old residing in randomly selected households in Guraghe zone, SNNPR, Ethiopia in 2018. Randomly selected school age children aged between 6 to 12 years of age from randomly sampled households with their parent or caregiver's pair were included in the study.

The sample size for the first objective was determined using single proportion formula with $95 \%$ confidence interval, critical value $(\mathrm{Z})$ as $1.96,5 \%$ marginal error $(\mathrm{d})$ and taking prevalence of inadequate dietary diversity among children from previous study as $(\mathrm{P}=47.6 \%$ and $\mathrm{p}=42.1 \%)$ from [15] using this formula:

$$
\begin{aligned}
& \mathrm{n}=\llbracket\left(\left(\mathrm{Z} \_2^{\wedge} \alpha\right) \rrbracket^{\wedge} 2(\mathrm{p}(1-\mathrm{P})) / \mathrm{d}^{\wedge} 2\right. \\
& \mathrm{n}=\llbracket((1.96)) \rrbracket^{\wedge} 2(0.476(1-0.476)) /\left[0.05 \rrbracket^{\wedge} 2\right. \\
& \mathrm{n} 1=382
\end{aligned}
$$

Thus, using prevalence of inadequate dietary diversity, as $42.1 \%$ in other provinces [15],

$$
\mathrm{n}=\llbracket\left((1.96) \rrbracket^{\wedge} 2(0.421(1-0.421)) /\left[0.05 \rrbracket^{\wedge} 2\right.\right.
$$

$\mathrm{n} 2=372$

Using the maximum sample size calculated from the first objective and design effect of 2 and $5 \%$ of non-response rate the final sample was as follows.

$$
\mathrm{n}=382 * 2=764
$$


$\mathrm{n}=764+(764 * 0.05)=803$

To select households with school age children, multi stage sampling was used. First, out of the total districts in the zone, five districts were randomly selected using simple random sampling (SRS) (namely Abeshegie, Cheha, Enamor Ena Enor, Mareko and Meskan districts). Then, from each woreda two kebeles were randomly selected from each district, using SRS (one from urban and one from rural to make more representative). Then, the calculated sample size were proportionally allocated to each districts and Kebeles (lower administrative unit below district) according to the expected number of school age children.

Households from respective kebeles were selected after identification of the geographic proximity center of the kebeles and then spinning pen was done. Then all households in the direction of a pen, were checked for school age children and interviewed till the ends of the Kebeles. When the desired sample size is not attained, another random spinning was done and households in that direction were interviewed. All randomly selected households with school age children from selected households were included in the study. Household when, child's parents were not volunteer to participate in the study and repeatedly closed households during the data collection period after revisits were not included in the study.

Data were collected by trained health extension workers through specified interview of the parent or caretakers of the children. Data was collected from selected households using a pretested interviewer administered questionnaire and more specific questions addressing dietary diversity. Wealth index was constructed using ownership status of certain household assets, cars and other assets. Translation of questionnaire to Amharic and Guragegna language questionnaire was done.

A ten food group Minimum Dietary Diversity for Women (MDD-W) tool adopted from Food and Nutrition Technical Assistance (FANTA) with the previous 24 hour dietary recall was used to assess a variety of food consumed. A study has validated that MDD-W had better performance over the seven food group dietary diversity score for infant and young children for school age children and older age groups [7]. It is also found to be better predictors for nutritional status of school age children [9, 16]. The data were collected from the child and their caregiver in a days where the days of interview and the previous consecutive days were not holidays, special days like marriage ceremony birth date or others. When there is unusual holidays or others ascertained from the respondent that respondent was not included in the survey as it can overestimate the dietary diversity.

Training of the data collectors was done before the data collection for two days. Close supervision was made by investigators and the assigned supervisors. Pre test was done on $10 \%$ of the sample, and necessary amendments were done accordingly. Feasibility of the study procedures, sampling, unclear questions and other sampling issues were evaluated and corrected. The daily collected data were checked for completeness and appropriate feedbacks were given for data collectors. Then the cross checked data were entered in to EpiData software version 3.01 and the data entry format was kept good via ranges, skip patterns and other issues. Double entry of the first $25 \%$ of the data was done by two independent data entry personnel. As the two entries does not show a significant difference, the remained data were entered by one data clerk. Some of the errors were corrected in comparison with the hard copy.

Cross checked data were entered into Epi data version 3.01 and exported to SPSS version 20 for analysis. To summarize and present the data; frequency, percentage, mean, median, Standard Deviation (SD) were interpreted. Adequate dietary diversity was categorized those children who consume at least five food groups using the 24 recall period out of the ten contextualized food groups [7]. Using the collected household assets, a five scale wealth index (category) was constructed using principal component analysis.

To assess determinants of low DD s among school age children, both bivariate and multivariate logistic regression was modelled using backward regression technique. The level of dietary adequacy was coded as zero and one (0-adequate dietary diversity and 1-inadequate dietary diversity) for those who consume above five and less than six food groups during the previous day respectively. Thus, both Bivariate and multivariate binary logistic regression will be employed. Crude Odds Ratio (COR) and adjusted odds Ratio (AOR) with 95\% Confidence interval were calculated. Those statistical associations with a $\mathrm{p}$ value less than 0.05 was declared as a statistically significant association. Model fitness was assessed using Hosmer and Lemeshow's test with $\mathrm{P}$ value greater than 0.05 as indicator for fitted regression model.

Formal ethical clearance was obtained from institutional Ethical review board of Wolkite University and letter of cooperation was taken from each selected district. As the study did not any confidential health outcome of the child, verbal informed assent was taken from the parents of children or caregiver before the interview after ethical approval for informed consent. All the information collected for this study is kept confidential in soft copy and hard copy in the hands of investigators. 


\section{RESULTS AND DISCUSSION}

In this almost greater than $95 \%$ response rate was observed, with almost all of the households $(84.6 \%)$ were led by fathers. But a significantly less number of fathers had formal literacy $(39.1 \%)$. Almost half the household relies on farming as the main source of living. While almost half (49.5) of care givers attended some forms of formal education in schools from primary to tertiary education level. More than half the school age children were aged between six to eight years, while the majority of children were males as shown in Table 1 .

Regarding the types of food groups eaten, 24 hour recall method was used to reduce the risks of memory lapse as time is extended. The tool included and interviewers probed the respondents for the contextualized lists of foods within each ten food groups. As the area is mainly relied on vegetables and fruits, they are consumed more frequently $(31.9 \%)$ as compared to cereals ad grains. More interesting vitamin A rich consumption is higher $(59.8 \%)$, egg $(64.8 \%)$ and pulses $(67.1 \%)$ which are important for the physical and metal growth of children. Additionally, milk products $(37.7 \%)$ and meat products $(69.6 \%)$ were also consumed by the school age children in the preceding day of the interview as shown in Table 2 . The mean dietary diversity score was 4.9 with a standard deviation of 1.42 . Overall more than half, 444 (58.3\%: $95 \%$ CI: $54.8 \%$ to $61.8 \%$ ) had an inadequate dietary diversity (less than five food groups) as shown in Figure 1.

Table 1. Child caregiver and household information in Southern Ethiopia

\begin{tabular}{cccc}
\hline Variables & Categories & No of respondents & Percentage \\
\hline \multirow{2}{*}{ Head of the house } & Father & 645 & 84.6 \\
Educational status & Caregivers & 117 & $15.4 \%$ \\
of the head & No formal education & 298 & 39.1 \\
Occupation & Attend formal education & 464 & 60.9 \\
& Farmer & 330 & 43.3 \\
Caregivers & Others* & 432 & 56.7 \\
Education & No formal education & 377 & 49.5 \\
Age of the child & Attend formal education & 385 & 50.5 \\
No of school age & 6-8 years & 456 & 59.8 \\
children within & 9-12 years & 306 & 40.2 \\
Household & One & 406 & 53.3 \\
Sex of child & Two & 283 & 37.1 \\
& Three and above & 70 & 9.6 \\
& Male & 447 & 58.7 \\
\hline
\end{tabular}

*Includes merchants, government employee, daily Laborer work and private worker

Table 2. A ten food group dietary diversity measure using 24 hour recall method

\begin{tabular}{cccc}
\hline Food Groups & Options & Frequency & Percentage \\
\hline Cereals and grains and food & Yes & 104 & 13.6 \\
made from these items & No & 658 & 86.4 \\
Fruits and vegetable & Yes & 243 & 31.9 \\
consumption & No & 519 & 68.1 \\
& Yes & 456 & 59.8 \\
Vitamin A rich plant foods & No & 306 & 40.2 \\
Other fruits and vegetables & Yes & 127 & 16.7 \\
& No & 635 & 83.3 \\
Egg & Yes & 494 & 64.8 \\
& No & 268 & 35.2 \\
Pulses/legumes & Yes & 511 & 67.1 \\
& No & 251 & 32.9 \\
Nuts and seeds & Yes & 511 & 67.1 \\
& No & 251 & 32.9 \\
Milk and milk products & Yes & 287 & 37.7 \\
& No & 475 & 62.3 \\
Meat and Meat product & Yes & 530 & 69.6 \\
Other meat and meat & No & 232 & 30.4 \\
products & Yes & 629 & 82.5 \\
& No & 133 & 17.5 \\
\hline
\end{tabular}

Int. J. Public Health Sci. Vol. 8, No. 2, June 2019: 211 - 218 


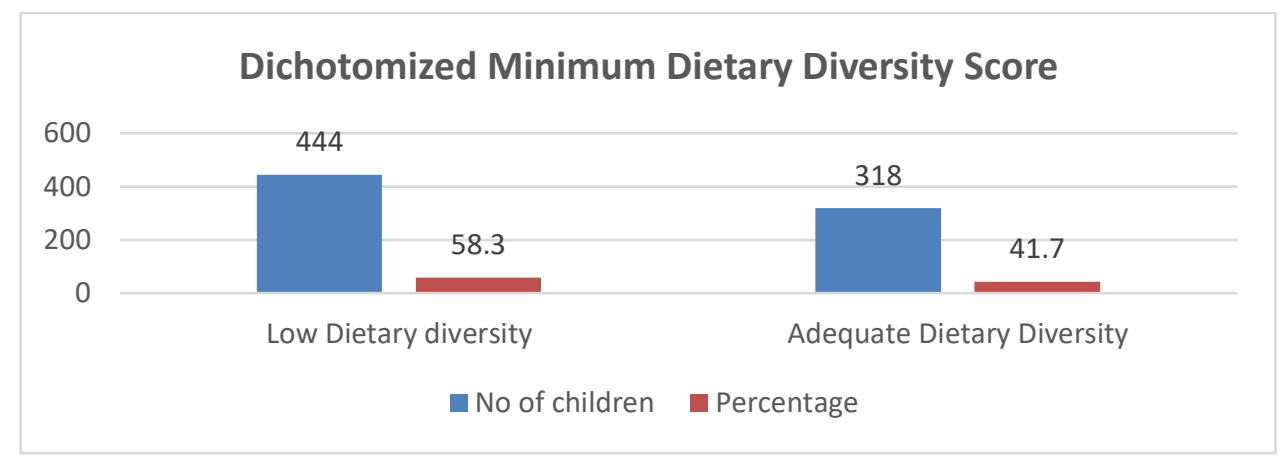

Figure 1. Dichotomized minimum dietary diversity score of school aged children

Female had shown lower dietary diversity score than males (COR=2.1, 95\% $\mathrm{CI}=1.6-2.8)$. Similarly, higher aged children had a lower DD score. The effects of the wealth index on DD score was mixed. Those from the lowest wealth quintile had shown significantly higher DD score as compared to highest, where as those of the second and the middle had lower DD score as compared from the highest wealth Quintile $(\mathrm{p}<0.05)$. Those children from extended family had reported a lower DD score as compared to family size below five members $(\mathrm{COR}=1.27,95 \% \mathrm{CI}=0.94-1.72)$. Children from father headed household had a higher dietary diversity score than female headed households (COR=2.4, 95\% CI=1.5-3.6). Those children in which household head and caregiver attended formal education had a higher DD score $(\mathrm{COR}=3.8,95 \% \mathrm{CI}=2.8-5.3)$ and $(\mathrm{COR}=5.6,95 \% \mathrm{CI}=4.0-7.6)$ respectively as shown in Table 3.

Table 3. Bivariate analysis showing factors associated with inadequate dietary diversity among school age children

\begin{tabular}{|c|c|c|c|c|c|}
\hline \multirow{2}{*}{ Variables } & & \multicolumn{2}{|c|}{ Dietary Diversity } & \multirow{2}{*}{$\begin{array}{c}\text { COR with } 95 \% \\
\text { CI }\end{array}$} & \multirow{2}{*}{$\begin{array}{c}\mathrm{P} \\
\text { value }\end{array}$} \\
\hline & & Inadequate & Adequate & & \\
\hline \multirow{2}{*}{ Sex of child } & Male & 228 & 219 & 1 & \\
\hline & Female & 216 & 99 & $2.1(1.6-2.8)$ & 0 \\
\hline \multirow[t]{2}{*}{ Child age } & Per each year increase in age & & & $1.1(1.0-1.2)$ & 0.028 \\
\hline & Lowest & 34 & 118 & $0.2(0.13-0.33)$ & 0 \\
\hline \multirow{4}{*}{ Wealth Index } & Second & 104 & 49 & $1.5(0.96-2.4)$ & 0.077 \\
\hline & Middle & 112 & 35 & $2.3(1.4-3.7)$ & 0.001 \\
\hline & Fourth & 91 & 43 & $1.5(0.94-2.4)$ & 0.091 \\
\hline & Highest & 103 & 73 & 1 & \\
\hline Educational level of & No formal education & 229 & 69 & $3.8(2.8-5.3)$ & 0 \\
\hline Head of Household & Attend formal education & 215 & 249 & 1 & \\
\hline \multirow{2}{*}{$\begin{array}{l}\text { Educational level of } \\
\text { caregiver }\end{array}$} & No formal education & 294 & 83 & $5.6(4.0-7.6)$ & 0 \\
\hline & Attend formal education & 150 & 235 & 1 & \\
\hline \multirow{2}{*}{$\begin{array}{c}\text { Occupational status } \\
\text { of head of } \\
\text { Household }\end{array}$} & farmer & 152 & 178 & 1 & \\
\hline & $\begin{array}{l}\text { Others (government, private, } \\
\text { merchant \& others) }\end{array}$ & 292 & 140 & $2.4(1.8-3.3)$ & 0 \\
\hline \multirow{2}{*}{ Head of household } & Father & 357 & 288 & 1 & \\
\hline & Others (mother, caregiver and other) & 87 & 30 & $2.4(1.5-3.6)$ & 0 \\
\hline \multirow{2}{*}{ Family size } & $<=4$ & 146 & 122 & 1 & \\
\hline & $>4$ & 298 & 196 & $1.27(0.94-1.72)$ & 0.118 \\
\hline
\end{tabular}

After adjusting for confounding the multivariate binary logistic regression, household head, caregiver's educational status, occupation of the household head, father/Female headed household and family size were found to be associated with DD score. Those children from extended family size had 1.3 times to have an inadequate DD level ( $\mathrm{AOR}=1.3,95 \% \mathrm{CI}=0.9-1.9)$. Similarly, those children from female headed households had 1.3 odds of having an inadequate DD level ( $\mathrm{AOR}=1.3,95 \% \mathrm{CI}=0.8-2.2)$. Children with household head did not attend formal education had 1.4 times risks of having lower DD score. Similarly, children living with uneducated caregiver had six fold more likely to have an inadequate DD level $(\mathrm{AOR}=6.7,95 \% \mathrm{CI}=4.1-11.0)$. This result can be seen in Table 4. 
Table 4. Multivariate analysis showing factors associated with inadequate dietary diversity among school age children

\begin{tabular}{|c|c|c|c|c|c|}
\hline \multirow{2}{*}{ Variables } & & \multicolumn{2}{|c|}{ Dietary Diversity } & \multirow{2}{*}{ AOR with $95 \% \mathrm{CI}$} & \multirow{2}{*}{$P$ value } \\
\hline & & Inadequate & Adequate & & \\
\hline \multirow{2}{*}{ Educational level of Head of Household } & No formal education & 229 & 69 & $1.4(0.9-2.3)$ & 0.186 \\
\hline & Attend formal education & 215 & 249 & 1 & \\
\hline \multirow{2}{*}{ Educational level of caregiver } & No formal education & 294 & 83 & $6.7(4.1-11.0)$ & 0 \\
\hline & Attend formal education & 150 & 235 & 1 & \\
\hline \multirow{2}{*}{ Occupational status of head of Household } & Farmer & 152 & 178 & 1 & \\
\hline & Others* & 292 & 140 & $4.7(3.2-6.8)$ & 0 \\
\hline \multirow{2}{*}{ Head of household } & Father & 357 & 288 & 1 & \\
\hline & Others $* *$ & 87 & 30 & $1.3(0.8-2.2)$ & 0.282 \\
\hline \multirow{2}{*}{ Family size } & $<=4$ & 146 & 122 & & \\
\hline & $>4$ & 298 & 196 & $1.3(0.9-1.9)$ & 0.103 \\
\hline
\end{tabular}

* Refers to public Employee, Private employee, Daily laborers and Merchant.

** Refers to mother, relatives, caregivers and other parents

This community based study assessed the dietary diversity and its predictors among school age children in Southern Ethiopia. Overall the mean Dietary diversity score of children was 4.6 foods were eaten in the preceding day of the interview which is below the minimum. Additionally 444 $(58.3 \%, 95 \% \mathrm{CI}=54.8 \%$ to $61.8 \%)$ found to have inadequate dietary diversity. In which cereals $(13.6 \%)$ were the least consumed food group followed by vegetables. Even if study by Habtamu and his colleges, targets young children the overall dietary quality is low (13\% had adequate variety food) [17].

In another study, grain has been the most frequently consumed food products in the northern part of Ethiopia. It is clear that the agricultural productivity of the country is diverse in that grains are not commonly grown food item in southern parts [17]. Study from Madagascar also depicted that almost 40 to $50 \%$ of children had diversified diet. Also foods like cereals and grains containing carbohydrates were the major sources of food while intake animal products like meat, egg and vitamin rich plant products was limited [15] which almost similar to our cases. Children should be encouraged on the intake of protein and vitamin rich food which is optimum for body growth and development $[11,16]$. Herrador Z. et. al. also depicted that there significant difference in consumption of Animal source foods $(64 \%$ versus $18 \%$ urban and rural respectively) [18] .

It is known that MDD is a proxy indicator for micronutrient adequacy measure in addition to the energy intake $[9,16]$. Even if the survey might pose social desirability bias on the types of food to be reported out of the ten food groups [7, 19], the overall measure of dietary adequacy is not satisfactory. A significantly higher proportion of children are consuming less diversified diet below the minimum requirement. Those food groups like fruits and vegetables, which are rich source of vitamin $\mathrm{A}, \mathrm{C}, \mathrm{K}$ and minerals, inadequate dietary intake might pose significant risks on the development of hidden hunger in addition to protein energy deficiency $[1,6,20]$. Low dietary diversity is also associated with increased burden of malnutrition $(\mathrm{P}=0.034)$ [11].

This will ultimately enhance the growth and developmental index deficits, including decreased school performances among children [1,21]. In this region an estimated $44.3 \%$ of children are affected by chronic malnutrition and a significantly high number of children (56\%) had some degree of anemia [22]. Diversifying the food that children feeding improves the nutritional status, prevents micronutrient deficiencies and improves linear growth of children [10].

In this study, children from extended family size had 1.3 times increased odds to have an inadequate DD level $(\mathrm{AOR}=1.3,95 \% \mathrm{CI}=0.9-1.9)$. Among the mechanisms of addressing global malnutrition is increasing household food accessibility, fair distribution of foods with the household members in accordance with their physiological requirement is crucial. Even if different cultural perceptions might affect this and increase their tendency to ignore such minor groups. In our study majority of households had at least two school aged children showing that the average family size is large. As the national level total fertility rate is about 4.6 [22], fair distribution of the food within the household had a paramount importance in addressing marginalized and nutritionally vulnerable groups.

Similarly, those children from female headed households had 1.3 odds of having an inadequate DD level $(\mathrm{AOR}=1.3,95 \% \mathrm{CI}=0.8-2.2)$. Children, in which fathers did not attend formal education $(\mathrm{AOR}=1.4, \mathrm{p}$ value $=0.186)$ and caregiver $(\mathrm{AOR}=6.7, \mathrm{p}$ value $<0.0001)$ had significantly lower diversified dietary intake. Study from North west Ethiopia among infant and young children also showed that women's involvement in decision making improved optimum dietary diversity (AOR=2.07 (1.02-4.20) [17]. Since majority of Ethiopian Households are headed and supported by father or others, those female headed households are more vulnerable to food insecurity, low income and wealth quintile. Additionally, similar 
studies also showed that educational status is key predictors of dietary diversity among children [15]. Similarly, study among Ethiopians showed that in male headed households, the intake of animal products is higher $(\mathrm{OR}=1.91, \mathrm{P}>0.05)[18]$.

It is evident that people's intention to feed varied foods to their children arises when the awareness and directive attitudes makes the person to practice that particular health behaviors. Thus, enhancing the educational level of caregivers, particularly person responsible for primary care of the child coupled with conducive environments like physical and economical access to food is important [23]. The above aforementioned results should be viewed in the light that respondents may have some biases that may overestimate the intake of vegetables, fruits, eggs and meat products. However, the study involved strict data collection method, analysis with larger sample size and the data was collected during the ends of the rainy season (pre harvest period). So this level of dietary diversity might be good and may show some improvements during the immediate post-harvest periods [12].

\section{CONCLUSION}

Food variety or dietary diversity of children in the study area was below average. Household head, caregiver's educational status, occupation of the household head, father/Female headed household and extended family size were predictors of low dietary diversity.

As low dietary diversity is common problem in the area, there should be an awareness creation through existing Health extension platform and back yard vegetation should be improved. Enhanced behavioral change schemes through community health agents, agricultural extension workers and volunteers there should be nutritional counseling for caregivers and the household as a whole, so as to improve and promote agricultural activities (farming variety of products) for the primary purpose of household consumptions.

\section{ACKNOWLEDGEMENTS}

Many thanks to study participants for their time and valuable information and Wolkite University, for funding this study.

\section{REFERENCES}

[1] Food and Agriculture organization of United nations (FAO). Schools-the beginning of the end of malnutrition. Zero Hunger 2018 [cited 2018 12/23].

[2] Zaida, H., S. Luis, G. Endalamaw, B. Antonio, G.-R. Rubén, et al., Micronutrient Deficiencies and Related Factors in School-Aged Children in Ethiopia: A Cross-Sectional Study in Libo Kemkem and Fogera Districts, Amhara Regional State. PLoS One, 2014.9 (12): p. e112858.

[3] Mekonnen H., Tadesse T. and Kisi T, Undernutrition and its Correlates among Rural Primary School Children of Fogera District, Northwest Ethiopia. J Nutr Disorders Ther, 2013. S12: p. 002.

[4] Mulugeta, A., F. Hagos, B. Stoecker, G. Kruseman, V. Linderhof, et al., Nutritional Status of Adolescent Girls from Rural Communities of Tigray, Northern. Ethiopia Ethiop.J.Health Dev. , 2009. 23 (1): p. 5-11.

[5] Ephrem, T., M. Jemal and M. Habtamu, Nutritional Status of School Children in Babile Town, Eastern Ethiopia. East African Journal of Health and Biomedical Sciences, 2017. 1 (2): p. 1-10

[6] World Health organization (WHO). Micronutrient Deficiencies: Vitamin A deficiecy. Micronutrient Deficiencies 2018 [cited 2018 23/12]; Available from: https://www.who.int/nutrition/topics/vad/en/.

[7] Caswell BL, Talegawkar SA, Siamusantu W, West KP Jr and P. AC., A 10-Food Group Dietary Diversity Score Outperforms a 7-Food Group Score in Characterizing Seasonal Variability and Micronutrient Adequacy in Rural Zambian Children. J Nutr. , 2018. 148 (1): p. 131-139.

[8] Sahar Hooshmand and Shobha A Udipi, Dietary Diversity and Nutritional Status of Urban Primary School Children from Iran and India. J Nutr Disorders Ther, 2013 (S12): p. 001.

[9] Wondafrash M, Huybregts L, Lachat C, Bouckaert KP and K. P., Dietary diversity predicts dietary quality regardless of season in 6-12-month-old infants in south-west Ethiopia. Public Health Nutr. , 2016. 19 (14): p. 2485-94.

[10] Mallard SR, Houghton LA, Filteau S, Chisenga M, Siame J, et al., Micronutrient Adequacy and Dietary Diversity Exert Positive and Distinct Effects on Linear Growth in Urban Zambian Infants. J Nutr. , 2016146 (10): p. 2093-2101.

[11] Olumakaiye M. F, Dietary Diversity as a Correlate of Undernutrition among School-Age Children in Southwestern Nigeria. Journal of Child Nutrition \& Management, 2013. 37 (1).

[12] Abdul-Razak Abizari , Fusta Azupogo, Miwako Nagasu, Noortje Creemers and Inge D. Brouwer, Seasonality affects dietary diversity of school-age children in northern Ghana. PLoS One, 2017. 12 (8): p. e0183206.

[13] Herrador Z, Perez-Formigo J, Sordo L, Gadisa E, Moreno J, et al., Low Dietary Diversity and Intake of Animal Source Foods among School Aged Children in Libo Kemkem and Fogera Districts, Ethiopia. PLoS One, 2015. 10 (7): p. e0133435. 
[14] Abizari AR, Azupogo F, Nagasu M, Creemers N and Brouwer ID, Seasonality affects dietary diversity of schoolage children in northern Ghana. PLoS One, 2017. 12 (8): p. e0183206.

[15] Rakotonirainy, N.H., V.r. Razafindratovo, R. RemonjaChitale, R. Rasoloarijaona, P. Piola, et al., Dietary diversity of 6- to 59-month-old children in rural areas of Moramanga and Morondava districts, Madagascar. PLoS One 2018. 13 (7): p. e0200235.

[16] Sié A, Tapsoba C, Dah C, Ouermi L, Zabre P, et al., Dietary diversity and nutritional status among children in rural Burkina Faso. Int Health. , 2018. 10 (3): p. 157-162.

[17] Habtamu Temesgen, Tebikew Yeneabat and Muluken Teshome, Dietary diversity and associated factors among children aged 6-23 months in Sinan Woreda, Northwest Ethiopia: a cross-sectional study. BMC Nutrition, 2018. 4 (5): p. 2-8.

[18] Herrador Z, Perez-Formigo J, Sordo L, Gadisa E, Moreno J, et al., Low Dietary Diversity and Intake of Animal Source Foods among School Aged Children in Libo Kemkem and Fogera Districts, Ethiopia. PLoS One, 2015. 10 (7): p. e0133435.

[19] Food and Agriculture Organization of the United Nations and USAID's Food and Nutrition Technical Assistance III Project (FANTA), Minimum Dietary Diversity For Women: A guide for Measurement, Nutrition, Editor 2016, FANTA: Rome, Italy. p. 1-8.

[20] Mary Arimond, Doris Wiesmann, Elodie Becquey, Alicia Carriquiry, Melissa Daniels, et al., Dietary Diversity as a Measure of the Micronutrient Adequacy of Women's Diets in Resource-Poor Areas: Summary of Results from Five Sites, in Technical Report, FANTA, Editor 2011, USAID: Washington, DC, USA.

[21] Zenebe M, Gebremedhin S, Henry CJ and Regassa N, School feeding program has resulted in improved dietary diversity, nutritional status and class attendance of school children. Ital J Pediatr., 2018. 44(1).

[22] EDHS, Ethiopia Demographic and Health Survey in Nutritional status of children I. international, Editor 2016, Central Statistical Agency Addis Ababa, Ethiopia .

[23] ACF, I., Food Security and Livelihood assessment; Apractical Guide, F.S.a. Livelihood, Editor 2010, ACF international. p. 92. 